\title{
Nonlinear Control of a Wheeled Mobile Robot with Nonholonomic Constraints
}

\author{
Chih-Fu Chang, Chin-I Huang and Li-Chen Fu \\ Electrical Engineering Department \\ National Taiwan University, Taiwan R.O.C. \\ D92921001@ntu.edu.tw
}

\begin{abstract}
This paper proposed a novel way to design and analysis nonlinear controllers to deal with the tracking problem of a wheeled mobile robots(WMR) with nonholonomic constraints. One of the nonlinear controllers is adopted to control the system with position and torque tracking requirements simultaneously. Another one is chosen to follow the path considering with position, torque and actuator dynamics by backstepping control. Both of feedback systems are shown to be exponentially stable via Laypunov stability analysis. In order to guarantee the highperformance operation of brushless DC motors (BLDCM) in such applications, the nonlinear model are accounted for increasing the precision actions through accuracy sketching nonlinear behaviours. The performance of controllers are verified through simulations.
\end{abstract}

Keywords-Wheeled mobile robot, nonlinear control, stability analysis, nonlinear system, dynamic model.

\section{Introduction}

In recent years, there has been enormous activity in the study of a class of nonholonomic systems, namely, wheeled mobile robot systems called. Specifically, due to the structure of the governing differentials equations of the underactuated nonlinear system, the regulation problem can't be solved via a smooth, time-invariant pure state feedback law due to the implications of Dixon's condition [1]. However, the models under investigation are basically kinematic ones. Recently, one method for dynamic models has been proposed in [3], which integrates a kinematic and a torque controller into the dynamic model of a nonholonomic mobile robot by using backstepping approach. Meng etc.[2] develop a fault tolerant adaptive control methodology switching among several controllers to maintain acceptable performance.

In a driect-drive servo system, the load is directly coupled to the rotor, and therefore, the torque generated by the motor is directly transmitted to the load. Hemant et al.[9] have designed an adaptive control methodology on BLDCM. The modeling problem of a BLDCM has been addressed by numerous authors ,e.g.,[10], [11], whose result are based on the assumption that the reluctance variations are negligible.

This paper is organized as follows. The problem formulation on this paper is introduced in Section 2. In Section 3, the nonlinear model of brushless DC motor is presented. System constrains, kinematics and dynamics, including rigid body and two wheel dynamics are addressed in Section 4. In Section 5, nonlinear controllers are developed. Simulations and discussions are proposed in Section 6. Finally, conclusions are drawn in Section 7.

\section{Problem formulation}

The nonlinear control problem for dynamic model of wheeled mobile robot with nonholonomic constraint and actuator dynamics is addressed in this paper. Figure 1 draws the conceptual diagram of the differential type of the wheeled mobile robot working in an indoor environment.

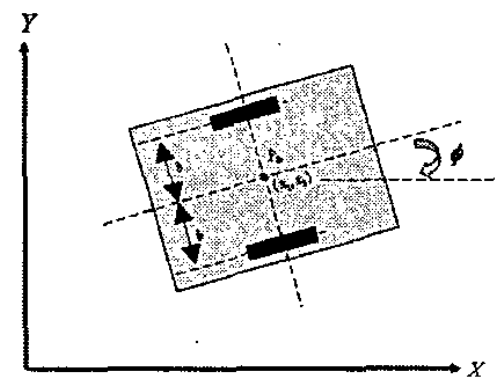

Figure 1 Schematic of the mobile robot

Where $b$ denotes the displacement from each of the driving wheels to the axis of symmetry. $d$ : the displacement from 
point $P_{0}$ to the process. $r$ : the radius of the driving wheels. $c:$ a constant equal to $r / 2 b \cdot m_{c}$ : the mass of the mobile robot without the driving wheels and the rotors of the wheels. $m_{w}$ : the moment of each driving wheel plus the rotor of its motor. $I_{c}$ : the moment of inertia of the mobile robot without the driving wheels and the rotors of the motors about a vertical axis through the interaction of the axis of symmetry with the driving wheel axis. $I_{w}$ : the moment of inertia of each driving wheel and the motor rotor about the wheel axis. $I_{m}$ : the moment of inertia of inertia of each driving wheel and the motor rotor about a wheel diameter.

There are two front driving wheels and a tail auxiliary wheel on the mobile robot. The vehicle is assumed to be the rigid body. Figure 2 depicts the control block diagram of the wheeled mobile robot control system.

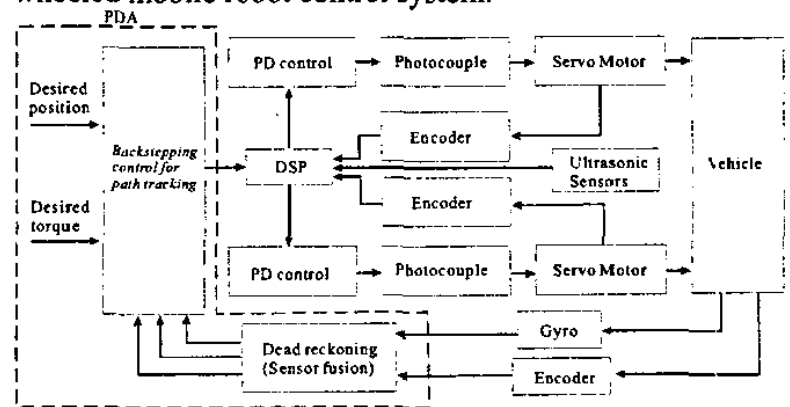

Figure 2 Block diagram of the wheeled mobile robot control system

The dead-reckoning system of the WMR is composed by odometries and rate-gyro. Odometry is a central part of almost all mobile robot navigation systems. The system's performance is decided by the system modeling; and controller design. The actual limitation is the assumption that WMR moves with a slow speed if the WMR's kinematic model is the main design consideration. Therefore, WMRs can not accelerate their speed easily and win very narrow bandwidth on the system's response with controllers designed by a kinematics model. However, more and more applications need fast and accuracy behavior on home, office or industrial field. For this reason, the purpose in this paper designs more accuracy and efficiency nonlinear controllers.

The physical control structure is divided into two parts. The first part is low level control. A TI DSP is the main processor to deal with control of both servo motors. The second part is high level control. Algorithms with more complex computations are executed in Personal Digital Assistant (PDA) system. The PDA is also equipped with the $\mathrm{A} / \mathrm{D}$ and $\mathrm{D} / \mathrm{A}$ module to facilitate communication via USB between the two parties.

\section{Nonlinear Model of a brushless DC motor}

Brushless DC motors are similar in performance and application to brush-type DC motors. Both have a speed vs. torque curve which is linear or nearly linear. The motors differ, however, in construction and method of commutation. A brush-type permanent magnet DC motor usually consists of an outer permanent magnet field and an inner rotating armature.

The servo controller and drive use the encoder feedback signal to continuously adjust the motor torque so that the desired position is maintained. This is referred to as a closed loop servo system. The electronics required to operate a brushless motor and "close the loop" are therefore more complex and expensive than micro-stepping or $\mathrm{dc}$ motor controls.

The nonlinear dynamic model is proposed by Neyram et. al.[10]. In the absence of magnetic saturation, it is convenient to formulate the dynamic behaviour of BLDCM as follows

$$
\&=f(L, \tau)+g(L) u(t)
$$

with

$$
\begin{aligned}
& f\left(L, \tau_{t}\right)=\left[\begin{array}{c}
\frac{3 n K_{e}}{2 J} L_{2}+\frac{3 n\left(L_{d}-L_{q}\right)}{2 J} L_{2} L_{3}-\tau_{t} \\
-\frac{n K_{e}}{L_{q}} L_{1}-\frac{R}{L_{q}} L_{2}-\frac{n L_{d}}{L_{q}} L_{1} L_{3} \\
-\frac{R}{L_{d}} L_{3}-\frac{n L_{q}}{L_{d}} L_{1} L_{2}
\end{array}\right], g(x)=\left[\begin{array}{ccc}
0 & \frac{1}{L_{q}} & 0 \\
0 & 0 & \frac{1}{L_{d}}
\end{array}\right]^{T} \\
& L(t)=\left[\begin{array}{lll}
L_{1} & L_{2} & L_{3}
\end{array}\right]^{T}, u(t)=\left[\begin{array}{l}
v_{q} \\
v_{d}
\end{array}\right] \\
& \quad=\left[\begin{array}{lll}
w & i_{q} & i_{d}
\end{array}\right]^{T}
\end{aligned}
$$

where $u$ denotes the actuator control command. $L \in R^{3}$, is the armature currents. $f(L, \tau): R^{3} \rightarrow R^{3} \quad$ and $g(x): R^{3} \rightarrow R^{3 \times 2}$ are smooth vector fields, which satisfies feedback linearizable property.

\section{Constraint Equation and SYSTEM Model OF WMR \\ 4.1 Constrain Equations}

There are three constrains. The first one is that the mobile robot can not move in a lateral direction, i.e.,

$$
x_{2} \cos \phi-x_{1} \sin \phi=0
$$

where $\left(x_{1}, x_{2}\right)$ the coordination of point is $P_{0}$ in the fixed reference coordinated frame $X-Y$, and $\phi$ is the heading angle of the mobile robot measured from $X$ axis. The other two constrains are that the two driving wheels roll without slipping:

$\alpha \cos \phi+\frac{\alpha}{2} \sin \phi+b \phi$ 


\section{$\not \alpha \cos \phi+\frac{\&}{2} \sin \phi-b \phi=r \theta_{2}^{\&}$}

(4)

where $\theta_{1}$ and $\theta_{2}$ are the angular positions of the two driving wheels, respectively. By using the techniques of differential geometry, it can be shown that, among the three constrains, two of them are nonholonomic and the third one is holonomic.

To obtain the holonomic constraint, we subtract equation (Eq. 3) from equation (Eq. 4).

$$
2 b \phi=r\left(\theta_{1}^{\&}-\theta_{2}\right)
$$

Integrating the above equation and properly chosen the initial condition of $\phi, \theta_{1}$ and $\theta_{2}$, we have

$$
\phi=c\left(\theta_{1}-\theta_{2}\right)
$$

which is clearly a holonomic constrain equation. The two nonholonomic equations are

$$
\begin{gathered}
\& \sin \phi-\cos \phi=0 \\
\text { (7) } \\
x_{1} \cos \phi+x_{2} \sin \phi=c b\left(\phi_{1}+\phi_{2}\right)
\end{gathered}
$$

The Lagrange equation is used to develop the system dynamic model. Kinematic nonholonomic constrained equation is derived in the matrix form as :

$$
A(q) \&=0
$$

where

$$
q=\left[x_{1}, x_{2}, \theta_{1}, \theta_{2}\right]^{r}, A(q)=\left[\begin{array}{cccc}
-\sin \phi & \cos \phi & 0 & 0 \\
-\cos \phi & -\sin \phi & c b & c b
\end{array}\right]
$$

$\phi$ denotes the heading angle, $b, c$ denote WMR constant parameters, and $\theta_{1}$ represents angular position of the wheel

The dynamic model is expressed as

$$
M(q) V(q, q) \&=E(q) \tau-A^{T}(q) \lambda
$$

where $A(q)$ is defined in Eq.(3), $M(q), V(q, \xi), E(q), \tau$ and $\lambda$ are well defined matrices according to the system dynamic equation.

\subsection{Kinematic Model}

The control variations of the WMR are composed of the velocity of the rigid body and the angular velocity of the heading angle. We derive the variations as

$$
\begin{gathered}
w=\frac{w_{r}+w_{l}}{2} \\
\mu=\frac{w_{r}-w_{l}}{w_{r}+w_{l}}
\end{gathered}
$$

where $w_{r}$ and $w_{t}$ denote the angular velocity of the wheeled. $w$ and $\mu$ can be used to control any vehicle movement. Thus, the slow-speed dynamics of the vehicle is expresses by

$$
\left[\begin{array}{r}
d x(t) / d t \\
d y(t) / d t \\
d \phi(t) / d t
\end{array}\right]=K\left[\begin{array}{l}
\cos \phi \cdot w \\
\sin \phi \cdot w \\
1 / b \cdot \mu \cdot w
\end{array}\right]
$$

(14)

where $\phi$ denotes the heading angle of WMR. $x, y$, the position relative to origin of WMR. $K_{1}$ : positive constant.

The Eq. (12) is performed with the following form as

$$
q=K_{1} J_{m}(q) u
$$

with

$$
\left[\begin{array}{l}
d x(t) / d t \\
d y(t) / d t \\
d \phi(t) / d t
\end{array}\right], J_{m}(q)=\left[\begin{array}{cc}
\cos \phi & 0 \\
\sin \phi & 0 \\
0 & 1
\end{array}\right], u=\left[\begin{array}{c}
r w \\
\mu w / b
\end{array}\right]
$$

According to Eq. (15), appropriate selections of $w_{\text {ave }}$ and $\mu$ will result in the required motion for the vehicle. Given the valves of $w_{\text {are }}$ and $\mu$, the right and left wheel speeds of the vehicle can be obtained from

$$
\begin{aligned}
& w_{r}=w(1+\mu) \\
& w_{l}=w(1-\mu)
\end{aligned}
$$

\subsection{Dynamic equations}

The Lagrange formulation is used to establish equations of motion for the mobile robot. The total kinematic energy of the mobile based and two wheels written explicitly

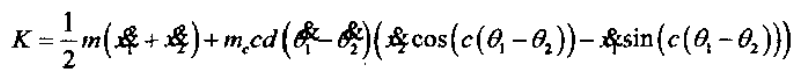

$$
\begin{aligned}
& +\frac{1}{2} I_{m}\left(\theta_{1}^{k}+\sigma_{2}^{*}\right)+\frac{1}{2} I_{c}^{2}\left(\phi_{1}^{\&}+\theta_{2}^{k}\right)^{2}
\end{aligned}
$$

where

$$
\begin{gathered}
m=m_{c}+m_{w} \\
I=I_{c}+2 m_{w} b^{2}+2 I_{m}
\end{gathered}
$$

Lagrange equations of motion for the nonholonomic mobile robot system are

$$
\frac{d}{d t}\left(\frac{\partial K}{\partial q_{i}}\right)-\frac{\partial K}{\partial q_{i}}=\tau_{i}-a_{1 i} \lambda_{1}-a_{2 i} \lambda_{2}, \mathrm{i}=1,2,3,4
$$

where $q_{i}$ is the generalized coordinate defined in equation (Eq. 9), $\tau_{i}$ is the generalized force, $a_{i j} s$ are the elements of matrix $A(q)$ in equation (Eq. 10), and $\lambda_{1}$ and $\lambda_{2}$ are the Lagrange multipliers. Substituting the total kinematic energy (Eq. 18) into Eq. 19, we obtain 


$$
\begin{aligned}
& m m_{c} d\left(c\left(\theta_{1}\right) \sin \left(c\left(\theta_{1}-\theta_{2}\right)\right)+c^{2}\left(\theta_{1}-\theta_{2}\right)^{2} \cos \left(c\left(\theta_{1}-\theta_{2}\right)\right)\right) \\
& =\lambda_{1} \sin \left(c\left(\theta_{1}-\theta_{2}\right)\right)+\lambda_{2} \cos \left(c\left(\theta_{1}-\theta_{2}\right)\right)
\end{aligned}
$$

$$
\begin{aligned}
& m+m_{c} d\left(c\left(\theta_{1} \theta_{2}\right) \cos \left(c\left(\theta_{1}-\theta_{2}\right)\right)-c^{2}\left(\theta_{1}^{k}-\theta_{2}\right)^{2} \sin \left(c\left(\theta_{1}-\theta_{2}\right)\right)\right)(21 \\
& =-\lambda_{1} \cos \left(c\left(\theta_{1}-\theta_{2}\right)\right)+\lambda_{2} \sin \left(c\left(\theta_{1}-\theta_{2}\right)\right) \\
& \text { ) }
\end{aligned}
$$

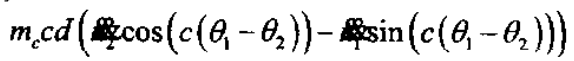

$$
\begin{aligned}
& +\left(I_{c}^{2}+I_{m}\right) \theta_{1}^{2} I_{c}^{2} \theta_{2}=\tau_{1}-c b \tau_{2}
\end{aligned}
$$

$$
\begin{aligned}
& -m_{c} c d\left(\frac{\pi}{2} \cos \left(c\left(\theta_{1}-\theta_{2}\right)\right)-\sin \left(c\left(\theta_{1}-\theta_{2}\right)\right)\right) \\
& -I_{c}^{2} \otimes_{1}^{\beta_{4}}\left(I_{c}^{2}+I_{m}\right) \tau_{2}-c b \tau_{2}
\end{aligned}
$$

where $\tau_{1}$ and $\tau_{2}$ are the torques acting on the two wheels.

These equations can be written in the matrix form

$$
M(q) q^{2} V(q, q) E(q) \tau-A^{T}(q) \lambda
$$

where $A(q)$ is defined in equation (10) and

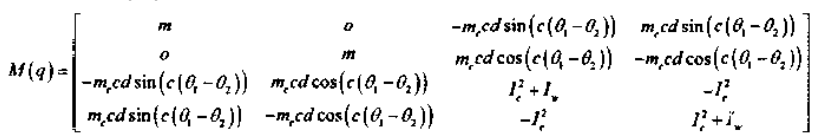

$$
\begin{gathered}
V(q, \phi)=\left[\begin{array}{c}
-m_{c} d\left(c\left(\theta_{1}-\theta_{2}\right)\right)^{2} \cos \left(c\left(\theta_{1}-\theta_{2}\right)\right) \\
-m_{c} d\left(c\left(\theta_{1}-\theta_{2}\right)\right)^{2} \sin \left(c\left(\theta_{1}-\theta_{2}\right)\right) \\
0 \\
0
\end{array}\right] \\
E(q)=\left[\begin{array}{ll}
0 & 0 \\
0 & 0 \\
1 & 0 \\
0 & 1
\end{array}\right], \tau=\left[\begin{array}{l}
\tau_{1} \\
\tau_{2}
\end{array}\right], \lambda=\left[\begin{array}{l}
\lambda_{1} \\
\lambda_{2}
\end{array}\right]
\end{gathered}
$$

The kinematic constraints are assumed to be expressed Eq. (9). With respect to the dynamics of mobile robot, Eq. (24), the following properties are known:

(a) $\left.x^{T}(q)-2 V(q, q)\right) x=0 \quad \forall x \in R^{n}$

(b) $\exists M_{m}, M_{M}$ s.t. $0<M_{m} \leq\|M(q)\| \leq M_{M}<\infty \quad \forall q \in R^{n}$

(c) $\exists V_{M}$ s.t. $\|V(q, x)\| \leq V_{M}\|x\| \forall q, x \in R^{n}$

which are exploited in driving the proposed control laws.

\section{Nonlineair controller design}

\subsection{Nonlinear Control without Actuator} dynamics

Lemma 1: Consider the mobile robot with nonlinear actuator dynamics expressed by Eq. (1),(9) and (24). The tracking error vector $e$

$$
e={ }^{w} R_{B} \psi_{0}
$$

with

$$
e=\left[e_{1}, e_{2}, e_{3}\right]^{T},{ }^{w} R_{B}=\left[\begin{array}{ccc}
\cos \phi & \sin \phi & 0 \\
-\sin \phi & \cos \phi & 0 \\
0 & 0 & 1
\end{array}\right], \phi_{0}=\left[\begin{array}{c}
x-x_{d} \\
y-y_{d} \\
\phi-\phi_{d}
\end{array}\right]
$$

Then the time derivative of the error

$$
\&=\left[\begin{array}{c}
w e_{2}-v+v_{r} \cos e_{3} \\
-w e_{1}+v_{r} \sin e_{3} \\
w_{r}-w
\end{array}\right]
$$

According to Barbalat's lemma, $\lim _{t \rightarrow \infty} \& \rightarrow 0$ when $q_{0} \in L^{\infty}$.

The nonlinear kinematic controller is proposed by Yutaka et al.[12].

Lemma 2: Let $e \in R^{3}$ and $e$ is bounded. Therefore, the control input $v_{c}$ is designed as bellow

$$
v_{c}=\left[\begin{array}{c}
v_{r} \cos e_{3}+k_{1} e_{1} \\
w_{r}+k_{2} v_{r} e_{2}+k_{3} v_{r} \sin e_{3}
\end{array}\right]
$$

where $k_{1}, k_{2}$ and $k_{3}$ denotes positive constant. $v_{r}$ : the desired velocity. Thus, the system is exponentially stable.

Theorem 1: Consider the system described by (24) with the control law given by the solution $\tau_{d}$ of the following algebraic equation

$$
\tau_{d}=E^{+}(q)\left(M q d q(q, q)+A(q) \lambda-K_{p} e-K_{d} \phi\right)
$$

with

$$
e=q-q_{d}, \&=\&-\Lambda e, s=\phi \&=\& \Lambda e
$$

where $\tau_{d}$ denotes the desired torque command, both $K_{p}$ and $K_{d}$ are positive definite diagonal matrices, and $q_{d} \in R^{n}$ and $\& \in R^{n}$ are the desired trajectories of mobile robot position and velocity variables. $q_{r} \in R^{n}$ and $q_{\gamma} \in R^{n}$ are the reference trajectories of mobile robot position and velocity variables, respectively. $E^{+}(q)=\left(E(q)^{T} E(q)\right)^{-1} E(q)^{T}$ is a pseudo inverse matrix. Then, the system is shown to be exponentially stable.

Proof: Appendix A

Corollary 1: From Lemma 1, Lemma 2 and Theorem 1 , the desired position is defined by Eq. (15) and transform to the control space

$$
\$=K_{2}\left(\&_{m}^{\&}(q) v_{c}+J_{m}(q) \&\right)
$$

Then the virtual control torque derived as

$$
\tau_{d}=E^{+}(q)\left(\bar{M}(q) K_{2}+\bar{V}(q, \bar{q}(u)) K_{1} v_{c}+A(q) \lambda-K_{p} e-K_{d} \&\right.
$$

According the proof of theorem 1, the system is exponentially stable.

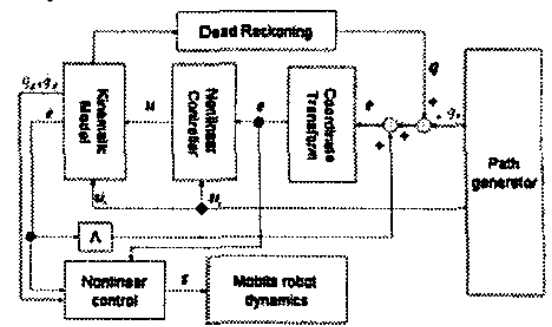


Figure 3 Nonlinear virtual torque control

Figure 3 is generated by Eq. (25) and Eq. (15). Actually, the implementation is suffering from solving the inverse torque problem when we neglect the nonlinear actuator dynamics. In this case, the linear relationships between output velocity, torque and voltage are assumed. Actually, the nonlinear relationships can be dinged out by experimental. Therefore, the following backstepping design methodology for system and actuator dynamics is adopted to derive a nonlinear position tracking controller for mobile robots in the case in which the system parameters are known.

\subsection{Nonlinear Control with Actuator dynamics}

Lemma 3: The angular position of the rotors and generalized displacement in articulation coordinates are related by

$$
\&=J_{r} u
$$

with

$$
\theta=\left[\begin{array}{l}
\theta_{1} \\
\theta_{2}
\end{array}\right], J_{r}=\left[\begin{array}{cc}
1 / 2 r & b \\
1 / 2 r & -b
\end{array}\right]
$$

where $u$ is defined by Eq. (15).

Relation between the joint's torques vector and the armature current vector is described by

$$
\tau=B K_{L} L
$$

where $B$ denotes an $2 \times 2$ positive definite constant diagonal matrix of gear ratios. $K_{L}$, an $2 \times 2$ positive definite constant diagonal matrix of actuator torque coefficients.

Corollary 2: Consider the Lemma 3 and Eq. (5), the heading angle of the WMR is represented as

$$
\phi=c\left[\begin{array}{ll}
1 & -1
\end{array}\right] J_{r} u
$$

Thus, by Barbalat's lemma, $\& 0$ as $t \rightarrow \infty$ when $\int u \in L^{\infty}$.

Definition 1: The actuator dynamics of the wheel is described by Eq.(1). After cascading two dynamic equations of the actuators, $f$ and $g$ are defined as

$$
f^{*}(L, \tau)=\operatorname{diag}\left(f_{i}\left(L_{i}, \tau_{i}\right)\right), g^{*}(L)=\operatorname{diag}\left(g_{i}\left(L_{i}\right)\right), i=1,2
$$

where $L_{i}$ is defined in Eq. (1)

Theorem 2: Consider the system described by Eq. (24), which satisfies Theorem 1 and Corollary 2, with the control law given by the solution $\tau_{d}$ of the following algebraic equation:

$$
\begin{aligned}
& \tau_{d}=E^{+}(q)\left(M(q)+V\left(q, d_{d}+A(q) \lambda-K_{t} e-K_{2} s\right)\right. \\
& u^{*}=g^{*+}\left(L_{d}\right)\left(-f^{*}\left(L_{d}, \tau_{d}\right)-K_{L}^{-1} B^{-1} s \Gamma^{-1}-K_{L}^{-1} B^{-1} K_{t} B K_{L} \ell\right)
\end{aligned}
$$

where $u^{*}=\left[v_{r}, v_{l}\right]^{\tau}$ depicts the input signal of the voltage command to BLDCMs. $K_{1}, K_{2}, K_{\tau}, \Gamma$ and $\Lambda$ are positive definite diagonal matrices, and $\mathcal{L} /=L-L_{d}, \mathcal{L} \in L^{2} \cap L^{x}$ is the bounded current error of mobile robot BLDCM's variables, respectively. $g^{*+}\left(L_{d}\right):$ a pseudo inverse matrix.
Proof: Appendix B

After the stability analysis, the system is shown exponentially stable.

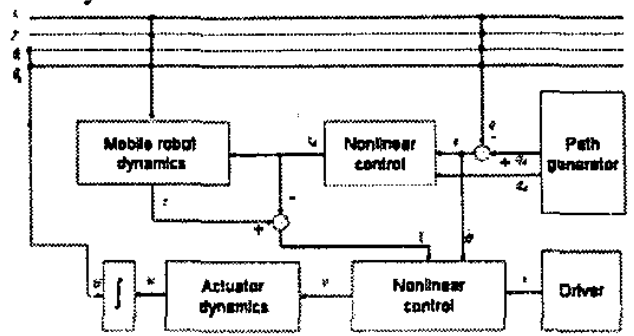

Figure 4 Block diagram of nonlinear backstepping control

\section{Simulation and discussion}

The Simulation has been performed to investigate the effectiveness of the proposed controllers and to obtain guidelines for experimentation. For this purpose, a realistic model of the experimental setup, a wheeled mobile robot, has been used. The system parameters were selected based upon their actual values and are given in Table 1 .

Table 1, The system parameters used in the simulations
\begin{tabular}{|c|c|c|}
\hline & Parameters & Value \\
\hline 1 & $b$ & $0.1 \mathrm{~m}$ \\
\hline 2 & $d$ & $0 \mathrm{~m}$ \\
\hline 3 & $r$ & $0.15 \mathrm{~m}$ \\
\hline 4 & $m_{c}$ & $50 \mathrm{~kg}$ \\
\hline 5 & $m_{w}$ & $5 \mathrm{~kg}$ \\
\hline 6 & $I_{c}$ & 0.45 \\
\hline 7 & $I_{w}$ & 0.125 \\
\hline 8 & $I_{m}$ & 0.325 \\
\hline
\end{tabular}

In first simulation, the unit step response is used for evaluation the performance of the nonlinear controller. The initial pose of the robot is $q(0)=\left[\begin{array}{llll}0 & 0 & 0 & 0\end{array}\right]^{T}$. When time arriyes $0.19 \mathrm{~s}$, a position of mobile robot changes $(t, 0)$ to $(t, 1)$ with $t$ is from $0.01 \mathrm{~s}$ to $1.2 \mathrm{~s}$. Observing figure 5 , no torque limitation is set, it reveals the good performance. 


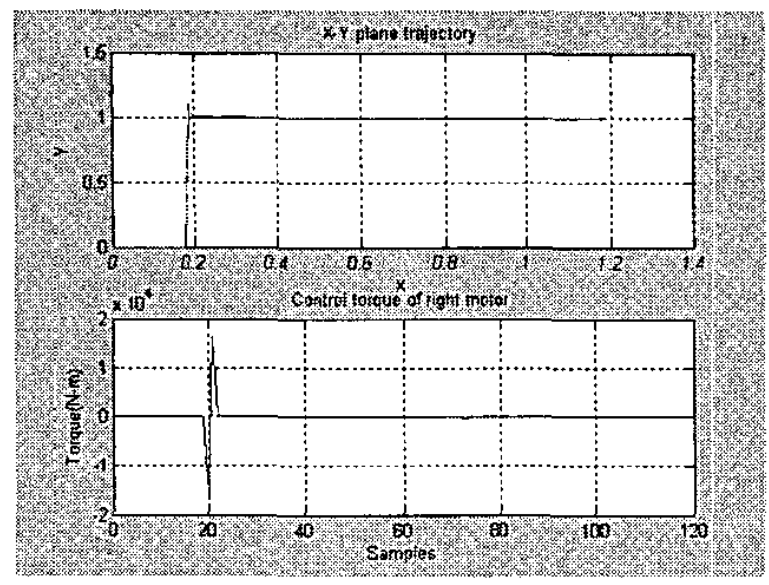

Figure 5 The drawing of unit step response

In second simulation, the initial pose of the path is $q_{r}=\left[\begin{array}{llll}0 & 0 & 0 & 0\end{array}\right]^{T}$, the initial state matrix with measurement error is given by $q(0)=\left[\begin{array}{llll}-0.5 & -0.5 & 0.01 & 0.01\end{array}\right]^{T}$. The default values of noise matrices are set by $N=\operatorname{diag}\left\{\begin{array}{llll}0.0001 & 0.0001 & 0.00001 & 0.00001\end{array}\right\}$, and the sampling interval is $T=0.01$ seconds. The WMR travels along a sinusoidal path with variational linear velocity such that $\Delta d=0.001 \mathrm{~m}$ and $\Delta \theta=0.001$. The robot is expected to navigate along a certain direction with desired trajectory.

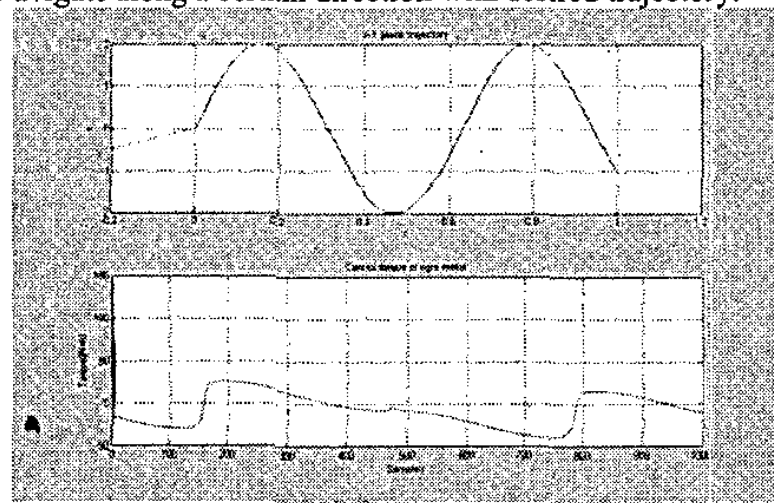

Figure 6 The tracking control result for sinusoidal path

In the simulations conducted for this paper, norlinear control strategies hybrid with different dynamical models has been followed. The advantage of this approach is that the stability can be proof in these condsider nonlinear models for a robot such as WMR. The control algorithms and the robot dynamics were all implemented using Maltab.

\section{Conclusions}

This paper has developed the nonlinear controllers in the case of dynamic model of a WMR with nonholonomic constraints and actuator dynamics. The analysis of the stability shows the system with proposed nonlinear controllers is exponentially stable. Moreover, simulation results have verified that the proposed nonlinear control is feasible and effective. Also, the presented methods offers one of the novel ways to design nonlinear controllers with nonholonomic constrains and actuator dynamics theoretically and systematically.

\section{APPENDIX A - Proof of Theorem 1}

Take (Eq. 28) into (Eq. 24), the system error function is derived as below

$$
M(q) \&\left(V\left(q, \&+K_{d}\right) s+K_{p} e=0\right.
$$

Choice a Lyapunov function $V=\frac{1}{2} s^{T} M(q) s+\frac{1}{2} e^{T} k_{p} e$ $L^{\&}=s^{T}\left(-\left(V(q, Q)+k_{d}\right) s-k_{p} e\right)+\frac{1}{2}\left(s^{T} \Lambda^{Q}(q) s\right)+(s-\Lambda e) k_{p} e$ $=-s^{T} k_{d} s-\Lambda e k_{p} e$

(a2) then, $\&<-\beta\|\|^{2}, \beta>0$ is exponentially stable.

APPENDIX B - Proof of Theorem 2

Substituting Eq. (34) into Eq. (24) yields the following error dynamics:

$$
M(q) \& 4\left(V(q, \&)+k_{1}\right) s+k_{2} e=\mathfrak{q}
$$

Note that the effect of mobile dynamics emerges as a nonzero $t /$, as the controller reduces to a passivity-based controller.

Let $v_{1}=\frac{1}{2} s^{T} M(q) s+\frac{1}{2} e^{T} k_{2} e$

(b2)

where $k_{2}$ is positive constant, then

$$
\stackrel{\&}{\&}=-s^{T} k_{2} s-e^{T} \Lambda e+\mathscr{L}
$$

(b3)

Using backstepping methodology

$v_{2}$, which is a Lyapunov function for the closed loop system, is selected as

$$
v_{2}=v_{1}+\frac{1}{2} \ell \% \Gamma
$$

where $\Gamma$ is positive definite

$$
\exists \alpha_{m}, \alpha_{M}>0 \rightarrow \alpha_{m}\|\|_{p}^{2}<v_{2}<\alpha_{M}\|\ell\|_{p}^{2}
$$

The control torque is selected as

$$
\tau_{d}=E^{+}(q)\left(M(q) \$+V(q, \mathbb{R})+A(q) \lambda-k_{1} e-k_{2} s\right)
$$

Take $v_{2}$ time derivative and substitute Eq. (b5) into Eq. (b4)

$$
\begin{aligned}
\&= & -s^{T} k_{1} s-e^{T} \Lambda k_{2} e \\
& +\left[s^{T} \Gamma^{-1}+B K_{L}(f(L, \tau)+g(L) u(t))\right] \Gamma e
\end{aligned}
$$

Choice control law

$$
u(t)=g^{*+}\left(L_{d}\right)\left(-f^{*}\left(L_{d}, \tau_{d}\right)-K_{L}^{-1} B^{-1} s \Gamma^{-1}-K_{L}^{-1} B^{-1} K_{t} t\right)
$$

Take control law into the above equation, one can rewrite

$$
\begin{aligned}
\& & =-s^{T} k_{1} s-e^{T} \Lambda k_{2} e-\left(f(L, \tau)+g(L) u(t)-E_{d}^{\&}\right) \Gamma \mathfrak{l} / \\
& =-s^{T} k_{1} s-e^{T} \Lambda k_{2} e-\mathfrak{\imath} \Gamma K_{\tau} \ell \%
\end{aligned}
$$




$$
\leq-\beta\|\widetilde{x}\|^{2}, \beta>0
$$

The system is exponentially stable in the Lyapunov sense.

\section{Reference}

[1] W.E. Dixon, M. S. de Queiroz,D.M.Dawson and T.J. Flynn," Adaptive Tracking and Regulation of a Wheeled Mobile Robot with Controller/Update Law Modularity",IEEE Transactions on Control Systems Technology, Vol.12,No. I,January 2004

[2]Meng Ji,Zhen Zhang,Gautam Biswas and Nilanjan Sarkar,"Hybrid Fault Adaptive Control of a Wheeled Mobile Robot",IEEE/ASME Tranactions on Mechatronics, Vol. 8,No. 2, June 2003

[3] Takanori Fukao, Hiroshi Nakagawa and Norihiki Adchi,"Adaptive Tracking Control of a Nonholonomic Mobile Robot",IEEE Tranactions on Robotics and Automation, Vol. 16, No.5, October 2000

[4] Zvi Shiller, and Yu-Rwei Gwo,"Dynamic Motion Planning of Autonomous Vehicles", IEEE Tranactions on Robotic and Automation, Vol.7, No. 2, April 1991

[5] Min-Soeng Kim, Jin-Ho Shin, Sun-Gi Hong and Ju-Jang Lee,"Design a robust adaptive dynamic controller for nonholonomic and disturbances", Mechatronics, Vol. 13, pp. $507-519,2003$

[6] H.G. Tanner, K.J. Kyriakopoulos, N.I. Krikelis,"Advanced agritural robots: kinematics and dynamics of multiple mobile manipulators handling nonrigid material", Computers and Electronics in Agriculture, Vol. 31, pp.91-105, 2001

[7] Zhong-Ping Jiang and Henk Nijmeijer,"Tracking Control of Mobile Robots: A Case Study in Backstepping",Automatica, Vol. 33, No. 7, pp. 13931399,1997

[8] Hemant Melkote and Farshad Khorrami,"Nonlinear Adaptive Control of Direct-Drive Bruless DC Motors and Applications to Robotic Manipulators", IEEE/ASME Tranactions on Mechatronics, Vol 4.No. 1, March 1999

[9] Neyram Hemati,James S.Thorp and Ming C.Leu,"Robust Nonlinear Control of Brushless dc Motor for Direct-Drive Robotic Applications", IEEE Tranactions on Industrial Electronics, Vol. 37, No. 6, Dec. 1990

[10] K.-H.Kim, I.-C.Baik,S.-K.Chung,M.-J.Youn,"Robust Speed Control of Brushless DC Motor Using Adaptive Inout-Output Linearisation Technique",IEE Proc. Electr. Power Appl.,Vol. 144,No. 6,Nov. 1997

[11] Neyram Hemati and Ming C.Leu,"A Complete Model Characterization of Brushless dc Motors", IEEE Tranaction on Industry Applications, Vol. 28, No. 1,Jan/Feb 1992

[12] Yutaka K., Yoshihiko K., Fumio M. and Tetsuo N.,"A Stable Tracking Control Method for a Non-Holonomic Mobile Robot", IEEE/RSJ Internal Workshop on
Intelligent Robots and Systems IROS pp. 1236-1241, Nov,1991 